\title{
New approaches to sportsman hernia; logical integration between surgery and muscles sport rehabilitation
}

\begin{abstract}
Abbreviations: PWD, posterior wall deficiency; SH, sportsman hernia; AP, athletes pubalgia; PBSI, pubic bone stress injury; SGI, sport groin injury; TEP, total extra peritoneal; AIWBE, active isometric weight bering exercises; CMSE, core muscles stabilizing exercise
\end{abstract}

\section{Editorial}

Sport Groin injuries are very common among professional Athletes, $40 \%$ of Sucker and Hokey players and $10 \%$ of all the Athletes, but lastly become common also amongst sport active people. In part is treated by conservative treatment and in part by Endoscopic surgery. Making the diagnosis for selecting the right treatment it is a challenge but lastly become more feasible after publishing the new guidelines for diagnosis and treatment of Sportsman Hernia. In some of the cases there is more complexes injury that will need combined Endoscopic surgery with conservative treatments.

In 1980 Gilmore was the first to describe the sport trauma to the groin and to the posterior wall and defined the pathology of the "Posterior Wall Deficiency" (PWD). He developed an anterior tissue repair with good early results but late high failure, had the honor by named the pathology "Gilmore's Groin"” but later on transferred to "Sportsman Hernia" (SH). On the other hand Sport Injury to the Pubic Bone, Symphysis Pubis, Rectus Muscles insertion and Adductor tendons insertions to the pubis is known for years. Different terminologies describe the injury like "Osteitis Pubis", "Rectal-Adductor syn." and later "Athletes Pubalgia" (AP) with the description of the pathology as "Pubic Bone Stress Injury" (PBSI). In spite of that, for many years no fully understanding and clear definition of the different pathologies lead to confusion on diagnosis and treatments. Lastly definition and differentiation of the pathologies was established and described in the "Guidelines for Diagnosis and treatment of Sportsman Hernia" as part of International Endo Hernia guidelines published at 2011 in the Journal Surgical Endoscopy ${ }^{1}$ and in the recent update. ${ }^{2}$

There are well defined two kinds of Sport Groin Injury (SGI); the Sportsman Hernia (SH) with the pathology of Posterior Wall Deficiency (PWD) and the Athletes Pubalgia (AP) with the pathology of Pubic Bone Stress Injury (PBSI). The different criteria for diagnosis and treatment of this two SGI were clearly described in the Guiltiness for Sportsman Hernia. In general, the symptoms of SH are more lateral in the groin, sharp pain radiated to the inner tight aspect with neurologic characteristic that appear during sport activities and relapse later on rest. Typically the pain will recurred whenever the athlete will return to sport activities. The symptoms of AP are more central, dull continues pain with inflammatory characteristic that continue after the sport activities stops and response to steroidal and NSAI drugs and physiotherapy. The two injuries have the same etiology and pathogenesis; Muscles strength was not build up appropriate for the level of sport activity performed. Bio-mechanical Imbalance of muscles force activity on the two opposite side of the pubic bones leads to the injuries. The background pathology for SH is sport trauma causing small and irreversible tears and weakening of the posterior

\author{
Volume 2 Issue I - 2015 \\ Moshe Dudai \\ Ramat Aviv Medical Center, Israel \\ Correspondence: Moshe Dudai, Hernia Excellence, Ramat \\ Aviv Medical Center, 3 Louis Pasture ST, Tel Aviv 68036, Israel, \\ Tel 972-77-706-0496, Cell +972-52-406-2727, Fax +972-77- \\ 706-2024, \\ Email info@hernia-excellence.com; dudai@netvision.net.il
}

Received: November 28, 2014 | Published: January 13, 2015

inguinal wall facial sheets and the adjusted tendons, the Transversalis Fascia and the Conjoint Tendon. This is leading to loss of the integrity of the posterior inguinal wall with prominent outwardly bulging during sport activities, causing strains of the nerves and pain. In the AP there is a stress injury with edema and inflammatory process in the Symphysis Pubis with posterior disc herniation, Pubic bone marrow edema and ligaments strain of the inserted tendons muscles; Rectus, Pyramidal and Adductors. There are clear and typical different findings by anamnesis and physical examination for $\mathrm{SH}$ versus AP. Recently imaging examinations demonstrate both typical pathologies; High resolution Dynamic US can demonstrate the SH PW Bulging pathology and MRI the AP PBSI inflammatory edematous pathology. ${ }^{3}$

According to the SH guiltiness and the recent update, surgery is superior to conservative treatment for SH -Level 1A of evidence-and ${ }^{4}$ Endoscopic Total Extra Peritoneal (TEP) retro-pubic and posterior wall mesh placement is the recommended treatment for $\mathrm{SH}$ with excellent results-Level $1 \mathrm{~A}$ of evidence. ${ }^{5,6}$ Specific surgical maneuvers adapted for the SH pathology have to be add to the mesh placement, including nerve entrapment release but categorically not to divide!. On the other hand only conservative treatment of Active Isometric Weight Bering Exercises (AIWBE) is recommended for AP when quit all recovered in 8-12 weeks -Level 1A of evidence, in no case Adductor Tenotomy can be done!.

In our study we found that good part of the athletes are presenting with findings of both types of SGI with different level of severity. SH is persistently bilateral Bill but PBSI expressed in different level of severity. We classified PBSI for grade 1 upto 5;1 for muscle involvement in one side of the PB without bone involvement and gradual up to 5 for SP and bone marrow edema with both sides muscles involvement. We realized that the SH was the first injury and because the athlete continue with extreme sport activities on top of the SH injury, others PBSI be caused. We worked in cooperation with physiotherapist and sport physiologist to build up a therapeutic program that is a combination of Endoscopic TEP posterior wall repair and reinforcement by mesh combined with Muscles Sport Rehabilitation (MSR) (AIWBE for peripheral muscles and Core 
Muscles Stabilizing Exercise CMSE). After examining different sequence combination of treatment we had found that the more effective and shorter recovery combination is starting with the surgery following by MSR. Performing first the repair of the loss injured posterior wall giving strength to the groin that act as an anchor for the active forced healing process of the muscles and tendons. Since January 2014 we had operated on 42Sportsman with diagnosis of Bill. SH \& PBSI grade 2-5. They were treated by BillEndo TEP, Nerve entrapment release and posterior wall reinforcement by mesh placement followed by MSR of IAWBE and CMSE. After 4-7weeks all were recovery to the level of previous sport activity and more!

This entity and treatment is applicable as well for non-athlete patients performing sport or heavy activities suffering from $\mathrm{Ch}$. Groin Pain without palpable Hernia. Athletes suffered from SGI have to be diagnosed correctly of the subtype injury, SH or AP, for selecting the surgical or the conservative way of treatment. Some of the athletes having both $\mathrm{SH}$ and $\mathrm{AP}$, in these sportsman combined treatments of Endoscopy with MSR has to be tailored according to the severity of the PBSI injuries. If the right treatment is selected the results are excellent and in shorter time.

\section{Acknowledgements}

None.

\section{Conflict of interest}

The author declares no conflict of interest.

\section{References}

1. Dudai M, Conde SM, Bittnerr R. Guideline recommendation for sportsman hernia-diagnosis and treatment. Surg Endosc. 2011;25:2834-2843.

2. Conde SM, Dudai M, Bittner R. Update of guideline recommendation for sportsman hernia-diagnosis and treatment. Surg Endosc. 2014;10.1007/ s00464-014-3917-8.

3. Paajanen $\mathrm{H}$, Brinck T, Hermunen $\mathrm{H}$, et al. Laparoscopic surgery for chronic groin pain in athletes is more effective than nonoperative treatment: a randomized clinical trial with magnetic resonance imaging of 60 patients with sportsman's hernia (athletic pubalgia). Surgery. 2011;150(1):99-107.

4. van Veen RN, de Baat P, Heijboer MP, et al. Successful endoscopic treatment of chronic groin pain in athletes. Surg Endosc. 2007;21(2):189193.

5. Paajanen H, Syvahuoko I, Airo I. Totally extraperitoneal endoscopic (TEP) treatment of sportsman's hernia. Surg Laparosc Endosc Percutan Tech. 2004;14(4):215-218.

6. Garvey JF. Computed tomography scan diagnosis of occult hernia. Hernia. 2012;16(3):307-314. 